Review

\title{
Interactive Association of Drugs Binding to Human Serum Albumin
}

\section{Feng Yang *, Yao Zhang and Hong Liang *}

State Key Laboratory Cultivation Base for the Chemistry and Molecular Engineering of Medicinal Resources, Ministry of Science and Technology of China, Guangxi Normal University, Guilin 541000, Guangxi, China; E-Mail: zhangyao1006@gmail.com

* Authors to whom correspondence should be addressed; E-Mails: jxyangfeng@gmail.com (F.Y.); hliang@mailbox.gxnu.edu.cn (H.L.); Tel./Fax: +86-773-212-0958 (F.Y. \& H.L.).

Received: 27 January 2014; in revised form: 17 February 2014 / Accepted: 18 February 2014 / Published: 27 February 2014

\begin{abstract}
Human serum albumin (HSA) is an abundant plasma protein, which attracts great interest in the pharmaceutical industry since it can bind a remarkable variety of drugs impacting their delivery and efficacy and ultimately altering the drug's pharmacokinetic and pharmacodynamic properties. Additionally, HSA is widely used in clinical settings as a drug delivery system due to its potential for improving targeting while decreasing the side effects of drugs. It is thus of great importance from the viewpoint of pharmaceutical sciences to clarify the structure, function, and properties of HSA-drug complexes. This review will succinctly outline the properties of binding site of drugs in IIA subdomain within the structure of HSA. We will also give an overview on the binding characterization of interactive association of drugs to human serum albumin that may potentially lead to significant clinical applications.
\end{abstract}

Keywords: human serum albumin; binding site; interactions of protein-ligands; interactive associations of drugs; structure of protein complex

\section{Introduction}

Human serum albumin (HSA), being the most abundant protein of blood plasma, has many important physiological functions. Among them, HSA regulates colloidal osmotic pressure, and transports numerous endogenous compounds such as fatty acids (FA), hormones, bile acids, amino acids, 
metals and toxic metabolites [1-6]. Additionally, there is a wide variety of drugs that are delivered to their targeting organs/tissues by binding with HSA [1,7-9]. Therefore, HSA not only protects the bound drugs against oxidation and influences the in vivo drug distribution, but also alters the pharmacokinetic and pharmacodynamic properties of drugs [7,10-13].

HSA is a single-chain, non-glycosylated polypeptide with a molecular weight of 66,500 Da containing 585 amino acids [1]. HSA is a helical protein with turns and extended loops, and resembles a heart shape, with approximate dimensions of $80 \times 80 \times 30 \AA[1,14]$. In general, drugs have two forms in circulation, namely, bound or unbound to plasma proteins. The unbound drugs can passively diffuse through the barriers constituted by endothelial cells into the organs where they are metabolized, biliary excretion or glomerular filtration in kidney $[7,15,16]$. The unbound drugs can also be distributed intracellularly via specific transport systems. Only free drug molecules interact with therapeutic targets to produce therapeutic effects [7,17]. In most instances, the unbound drug concentration of drug in the tissue depends on the unbound drug concentration in the plasma [7]. Thus, HSA-drug interactions are an important factor to understand the pharmacokinetics and pharmacological effects of drugs [18-22].

The interactions between HSA and ligands have been extensively studied for several decades using a variety of methods [23-36]. However, since HSA is a flexible macromolecule featuring sophisticated binding sites for drugs, a detailed study of this system has been difficult to achieve. In fact, despite various HSA crystals were obtained several decades ago, the structure of this protein was difficult to elucidate due to the X-ray structure obtained from a crystal has low resolution [37-39]. It was not until 1992 that the HSA structure was really resolved by Carter et al. [40]. Furthermore, the structure of high resolution HSA complex was resolved in 1998 by curry et al. for the first time [41]. Subsequently, the structures of HSA forming complexes with other compounds have been popular and extensively studied by other groups, especially the group of Curry [42-66]. The structure of HSA reveals the presence of three domains, namely domains I (residues 1-195), II (196-383) and III (384-585), which, as predicted from amino acid sequence comparison, are not only topologically identical, but they also have similar three-dimensional structures $[14,40]$. The three domains are further divided into sub-domains A and B (Figure 1A). HSA contains 35 cysteine residues, and all of them except one, Cys34 (in domain I), are involved in disulfide bonds stabilizing the structure of HSA [14].

A fundamental characteristic of HSA is its surprising capacity to bind a large variety of drugs [1]. Taking into account the high concentration of HSA in plasma, the binding affinity of drugs to HSA is an important factor to be considered when designing and developing new drugs [67-71]. In addition, the interactive association of drugs that bind simultaneously to HSA can change the HSA binding behavior and potentially modulate the final therapeutic efficiency of the drugs [72]. Recent reported structures of HSA-ligands complexes not only clearly demonstrate the location of different drug binding sites on HSA, but they also revealed how several drugs interact with HSA [53,73-76]. Such knowledge on the binding properties of drugs to HSA is an important issue when analyzing the mechanisms affecting the pharmacological effects of these compounds. In this work, we focused on the nature of the HSA binding sites and the mechanisms of interactive association of drugs to HSA based on the available structural evidence found on HSA-drug complexes. Additionally, we highlight possible strategies that could be implemented to improve HSA-based delivery systems. 
Figure 1. The overall structures of human serum albumin (HSA) (A) and HSA-fatty acids (FA) (B). Carbon atoms of FA molecule are shown in yellow; oxygen in red; nitrogen in blue; domain IA, in red; domain IB, in green; domain IIA, in blue; domain IIB, in magenta; domain IIIA, in cyan; domain IIIB, in grey.

(A)

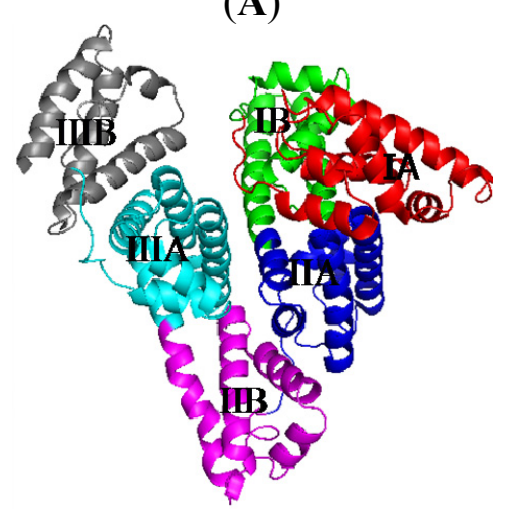

(B)

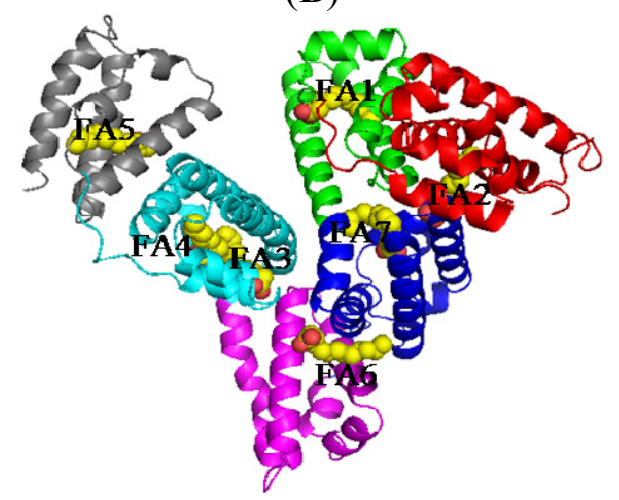

\section{Binding Site of Drugs in IIA Subdomain of HSA}

Since HSA has a limited number of high-affinity binding sites, detailed molecular information about these sites could be helpful in the assessment of cooperative effects during the binding of other drugs or endogenous ligands. Furthermore, the structural information of both high and low-affinity binding sites is useful when designing new drugs whether the aim is to avoid binding to HSA, or to make use of its depot function [67]. Therefore, the studies of drug binding sites on HSA have been important in the past and they keep being popular to date. Sudlow et al. did pioneering studies in this field by using a fluorescent probe displacement method in 1975 and 1976 [77,78]. With this method they showed through a screening, that there are two specific drug binding sites on HSA, namely, site I (also called the warfarin binding site) and site II (the benzodiazepine binding site). These excellent studies promoted the topology analysis and mapping of the drug binding sites on HSA. Using albumin fragments derived from pepsin and trypsin digestions, Bos et al. proposed that sites I and II are located in domains II and III, respectively [79,80]. Current crystallographic studies have proved that the majority of drugs bind to the above two main binding sites [53,74-76]. Certainly, these findings do not exclude the presence of other special binding sites on HSA [41,44,46,65,68]. However, in this review we only describe the main one binding site of HSA, namely, site I (IIA subdomain) owing to the following reasons. First of all, in the presence of fatty acids (FA), drugs bind preferentially to IB and IIA subdomain because IIIA subdomain is the strong binding site for fatty acids, thus fatty acids occupy this site inhibiting other drugs to bind here [81,82] (Figure 1B); In contrast, FA weakly bind to IIA subdomain, and is usually replaced by drugs. Additionally, interactive association of drugs binding to HSA usually occurs in IIA subdomain because site I is a big hydrophobic cavity that is possible to hold several drugs at the same time.

Site 1 is a preformed binding pocket within the core of subdomain IIA, which comprises six helices of the subdomain and a loop-helix feature (residues 148-154) contributed by subdomain IB. The interior of the pocket is hydrophobic, predominantly delimited by residues Trp214, Leu219, Phe223, Leu238, His242, Leu260, Ile264, Ser287, Ile290, and Ala291. However, it also contains also 
two clusters of polar residues, an inner cluster of residues toward the bottom of the pocket (Tyr150, His242, Arg257) and an outer cluster at the pocket entrance composed of Lys195, Lys199, Arg218, and Arg222 (Figure 2A). The large binding cavity is comprised of a central zone from which three distinct compartments extend. The distal end of the pocket is divided by residue Leu264 into left and right hydrophobic sub-chambers, whereas a third sub-chamber, delineated by residues Phe211, Trp214, Ala215, Leu238, as well as aliphatic portions of residues Lys199 and Arg218, protrudes from the front of the pocket (Figure 2A). Upon FA binding, residue Tyr150 from subdomain IB moves to interact with the carboxylate moiety of the lipid bound to a site that straddles domains I and II (fatty acid site FA2). This interaction helps to drive the relative rotation of domains I and II and has a large impact on only one side of drug site I. Extensive rearrangement of the H-bond network occurs, involving residues Tyr150, Glu153, Gln196, His242, Arg257, and His288, which opens a solvent channel between Tyr150 and Gln196, thus increasing the pocket volume and altering the distribution of polarity: the inner polar cluster is disrupted and partially neutralized by FA binding, whereas residue His 242 is relatively unaffected (Figure $2 \mathrm{~B}$ ).

Figure 2. The binding environment of ligand site in IIA subdomain of HSA. (A) Site I; (B) Site I in HSA-FA. Domain IB, in green; domain IIA, in blue; Dashed line (red), hydrogen bond.

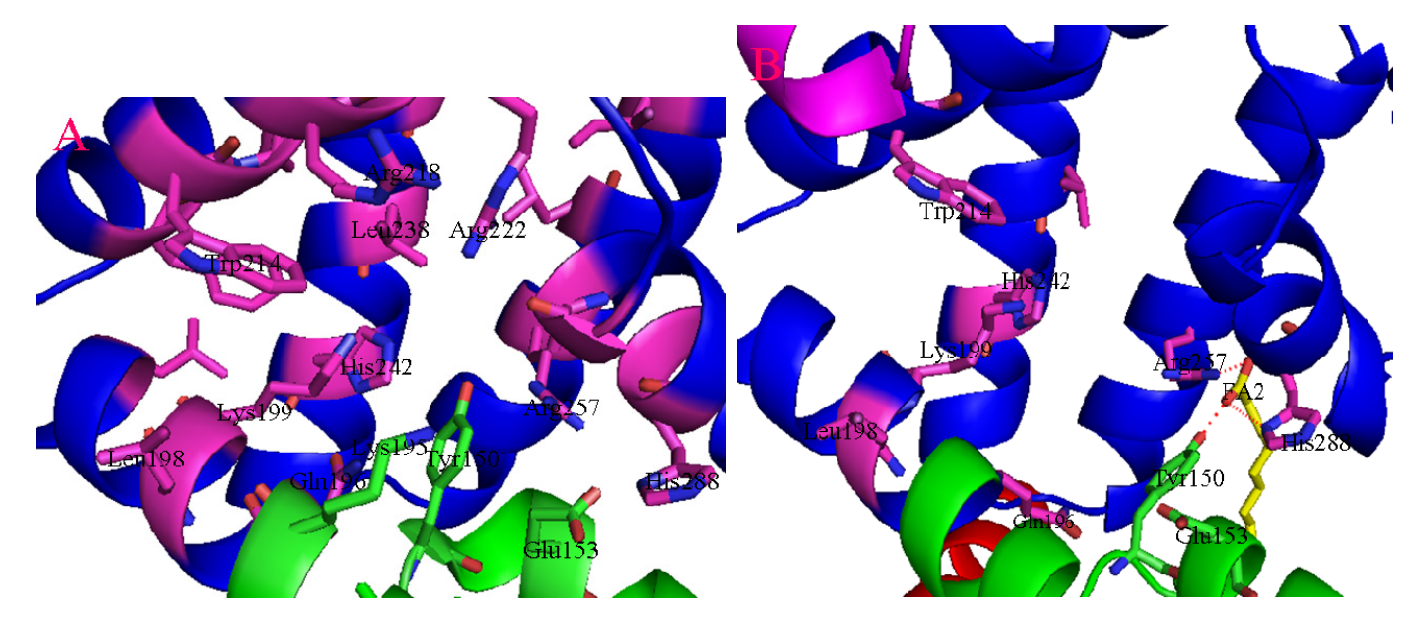

\section{Interactive Association of Drug-Drug with HSA}

Drug-drug interactions at the protein-binding level can be useful for therapeutic purposes because alteration in protein binding may change the volume of distribution, clearance, and elimination of a drug and may modulate its therapeutic effect $[8,72,83]$. It was the first structures of drug-drug interactions with HSA resolved by Curry et al., which provided a high value template for exploring drug-drug interactions [53]. Curry et al. initially suggested that indomethacin (IMN) would co-bind with some other site I specific compounds such as azapropazone (AZP), oxyphenbutazone, phenylbutazone (PLZ), 3,5-diiodosalicylic acid and tri-iodobenzoic acid by superposing the HSA-FA-IMN structure with those for other HSA-FA-drug complexes. To test this idea, PLZ-IMN and AZP-IMN double-drug were soaking into HSA-FA crystals. As a matter of fact, their experiment fit well with their hypothesis. The structures revealed that IMN and either AZP or PLZ do not displace one another from HSA, but simultaneously co-exist in the IIA subdomain (Figure 3A,B). Interestingly, the two drugs are 
slightly shifted if we compared them with their positions in the corresponding single drug complexes presumably as a result of drug-drug contacts [53]. The most striking effect of co-binding of these two drugs is the concerted rearrangement of Arg218 and Arg222, the principal effect of which is to substitute Arg222 instead of Arg218 as a binding partner for the carbonyl group of PLZ [53]. Additionally, Huang et al. think the presence of fatty acid is necessary for drugs' co-binding in IIA subdomain due to fatty acid can induce conformational changes of HSA to create new sub-site [56]. For example, they observed that two compounds (3'-azido-3'-deoxythymidine (AZT) and FA) coexist in the IIA subdomain [56]. HSA-FA-AZT structure revealed that AZT does not displace FA7, but moves to a new subsite of the IIA subdomain which is different from the subsite of IMN (Figure 3A-C). FA7 is still at the centre of IIA subdomain, and forms a hydrogen bond with AZT (Figure 3C). This new AZT subsite is close to subdomain IB and is besieged by hydrophilic and polar amino acids, including Glu153, Ser192, Lys195, Gln196 from subdomain IB and Lys199, His242, Arg257, Glu292 from site I [56]. Interestingly, from the HSA-FA-AZT-salicylic acid complex structure, Huang et al. [56] also observed the coexistence of AZT with another drug (salicylic acid (SA)) in IIA subdomain. SA replaces FA7 and bind at the center of IIA subdomain; AZT still remains in its original position, but AZT has no contact with SA although AZT forms hydrogen bonds with Glu153 and Arg257 (Figure 3C,D). Therefore, Huang et al. suggest that site I of HSA can be divided into three non-overlapped subsites: a SA subsite, an IMN subsite and an AZT subsite [56]. Unfortunately, the above structures only showed that the binding mode and binding site of drugs can affect each other when they co-bind to the IIA subdomain, but how is their binding affinity? Namely, is the binding affinity of drugs increasing, decreasing or do not influence each other? Fluorescence quenching demonstrated that the binding affinity of IMN to HSA is stronger than that of cinnamic acid (CA). The structures of the corresponding HSA complexes revealed that IMN and CA have a common binding site in the IIA subdomain. What would happen if IMN and CA interact with HSA at the same time? The structure revealed that IMN is at the center of the binding site, which is different form binding properties of IMN in PLZ-IMN and AZP-IMN interactions, but CA reposition itself through an alternative binding in a new subsite (Figure 3A,B,E). However, both the binding modes and binding affinities of these drugs suffer changes. In the presence of IMN, the binding affinity of HSA for CA decreased about five times compared with the same measure in the absence of IMN (Table 1). On the other hand, the binding affinity of IMN to HSA in the presence of CA was enhanced 1.4 times compared to the same measure in the absence of CA. These differences are attributable to their corresponding interaction forces with HSA [74]. CA just forms one hydrogen bond with IMN, contacting fewer residues of HSA than CA without IMN. However, IMN not only forms hydrogen bonds and salt bonds with HSA, but also interacts with CA. Obviously, CA is helpful in the binding of IMN to the IIA subdomain because Gibbs free energy $(\Delta G)$ of IMN interacting with HSA-CA is less than that of IMN with HSA. Although IMN inhibits CA to bind IIA subdomain to some extent, IMN supports lamivudine (LMD) to bind with IIA subdomain in that $\Delta G$ of LMD directly binding to HSA is higher than that of LMD interacting with HSA-IMN (Table 1), which improves about two times the binding affinity of HSA for LMD compared to the same measure in the absence of IMN [64]. LMD rotates approximately $20^{\circ}$ compared to LMD in HSA-FA-LMD structure, interacting with IMN (Figure 3F). To sum up, binding of a drug to HSA sometimes influences simultaneous binding of other drugs, thus their binding characterization may be affected by each other. 
Table 1. The binding constants $(K)$ and free energy change $(\Delta G)$ of HSA and HSA complexes for drugs.

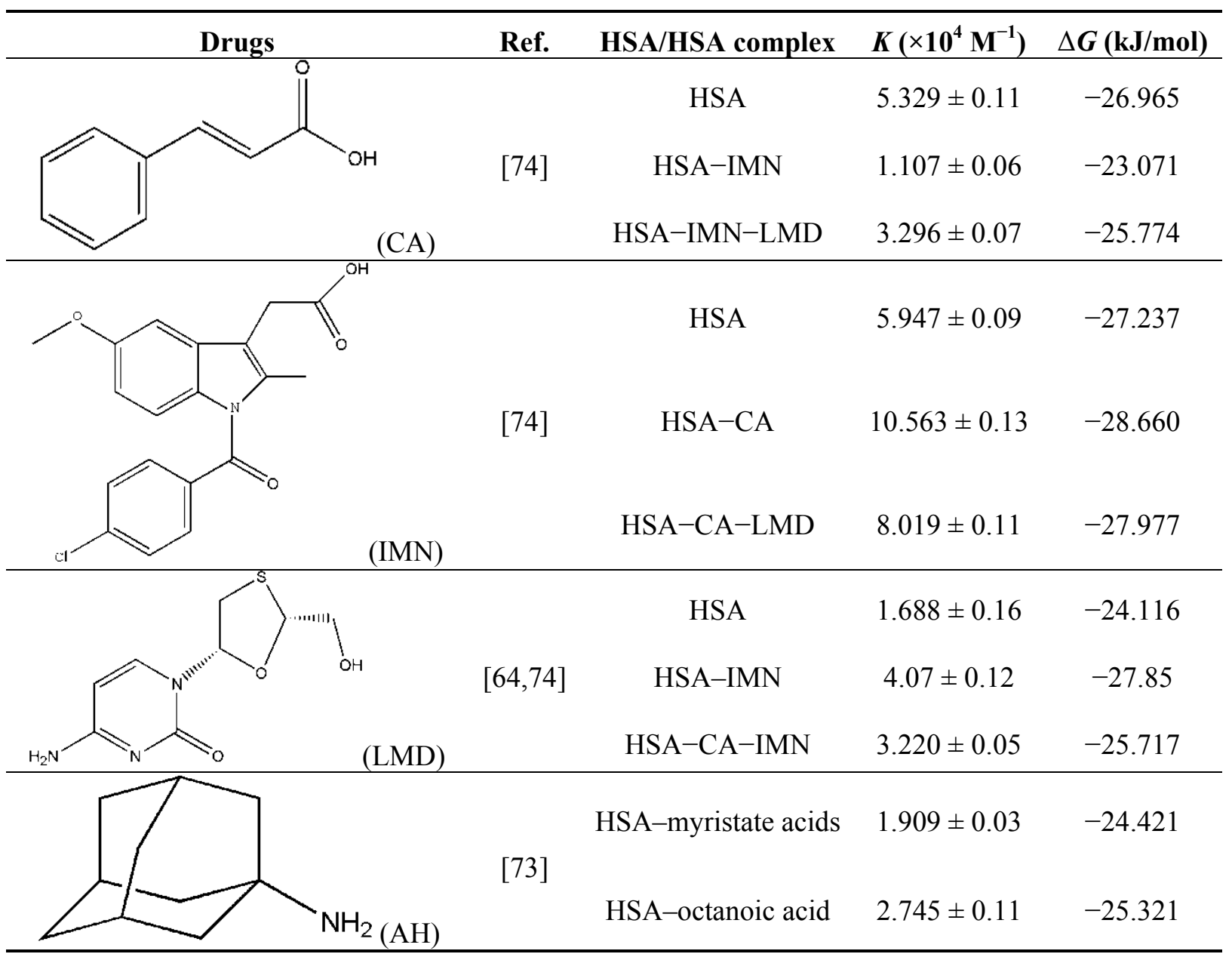

\section{Interactive Association of Drug-Drug-Drug with HSA}

In clinical practice, several drugs are often used to treat the disease synergistically, the binding strength of drugs to HSA plays a role in final therapeutic efficiency of the drugs [11]. Therefore, maybe we play optimal efficiency of drugs by interactions of drugs to regulate their binding affinity. The analysis of HSA structures has revealed that two drugs may co-bind the large hydrophobic cavity of the IIA subdomain (Figure 3). However, can several drugs co-bind this subdomain at the same time? To confirm the hypothesis, Yang et al. superposed the three structures corresponding to the complexes HSA-FA-IMN, HSA-FA-CA and HSA-FA-AZT, which showed that the three drugs have no overlap as they bind different positions of the IIA subdomain, which suggest that the three drugs could coexist simultaneously in the IIA subdomain [74]. As a matter of fact, the structure of the HSA-FA-CA-IMN-LMD complex revealed that the three drugs bind in the same binding site of the IIA subdomain [74]. Surprisingly, LMD does not bind to new subsite like AZT, but is at the center of IIA subdomain (Figure 4A). In fact, the binding affinity of CA and IMN to HSA are great stronger than that of LMD to HSA, but CA and IMN makes reposition (Figure 4B,C). The binding affinity of HSA for CA is a little affected by the presence or absence of IMN and LMD because CA moves to new subsite where AZT binds, forming hydrogen bonds with Glu153 and Ser192 (Figure 4A,C). However, the binding affinity of IMN to HSA is weaker than that of IMN in the presence of CA or CA 
and LMD. Obviously, the presence of the other drugs strengthens IMN to bind with IIA subdomain. Upon coexisting with other drugs, IMN is also easier to bind with HSA because the Gibbs free energy of IMN interacting with HSA is higher than that of IMN to HSA in the presence of other drugs.

Figure 3. Structural basis of drug-drug co-binding to subdomain IIA of HSA. (A) azapropazone (AZP)-indomethacin (IMN); (B) phenylbutazone (PLZ)-IMN; (C) FA-3'-azido-3'-deoxythymidine (AZT); (D) salicylic acid (SA)-AZT; (E) IMN-cinnamic acid (CA); (F) Comparison of binding mode of lamivudine (LMD) to IIA subdomain of HSA in the presence/absence of IMN. Dashed line (red), hydrogen bond.

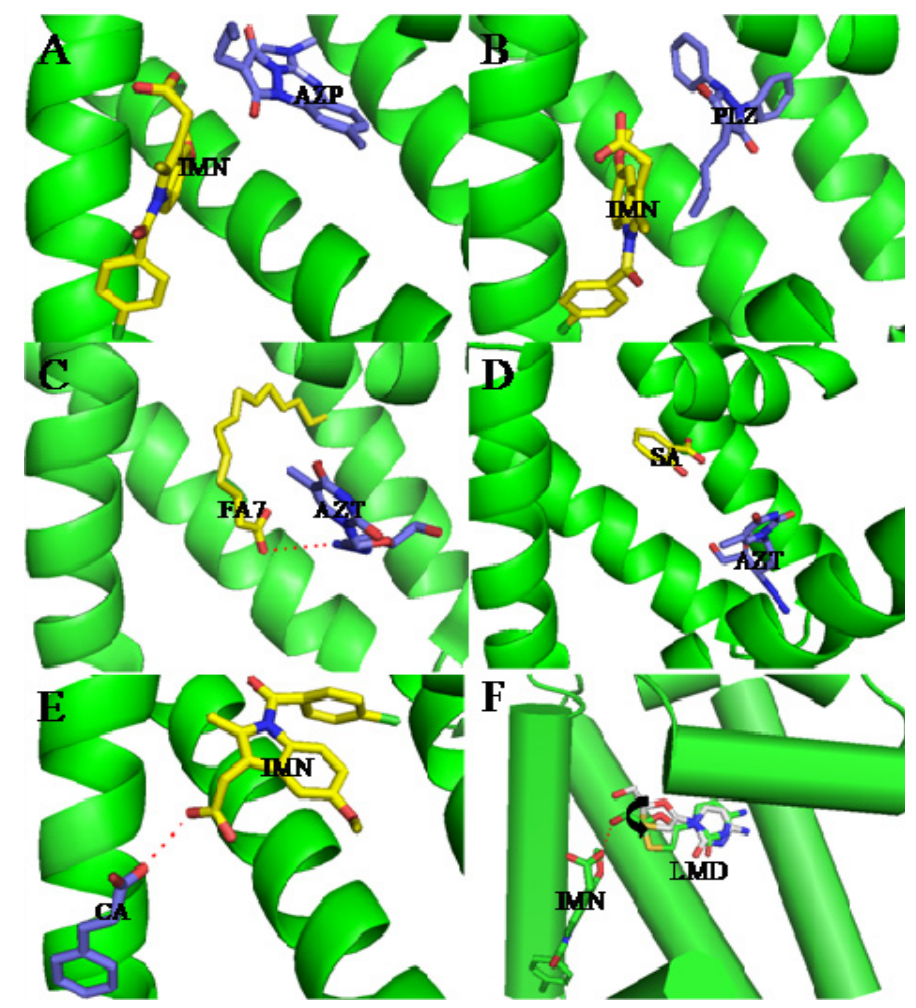

Figure 4. (A) Structural basis of IMN-CA-LMD co-binding to subdomain IIA of HAS; (B) the reposition route of IMN in the presence of CA and LMD; (C) the reposition route of CA in the presence of IMN and IMN-LMD. Dashed line (red), hydrogen bond.

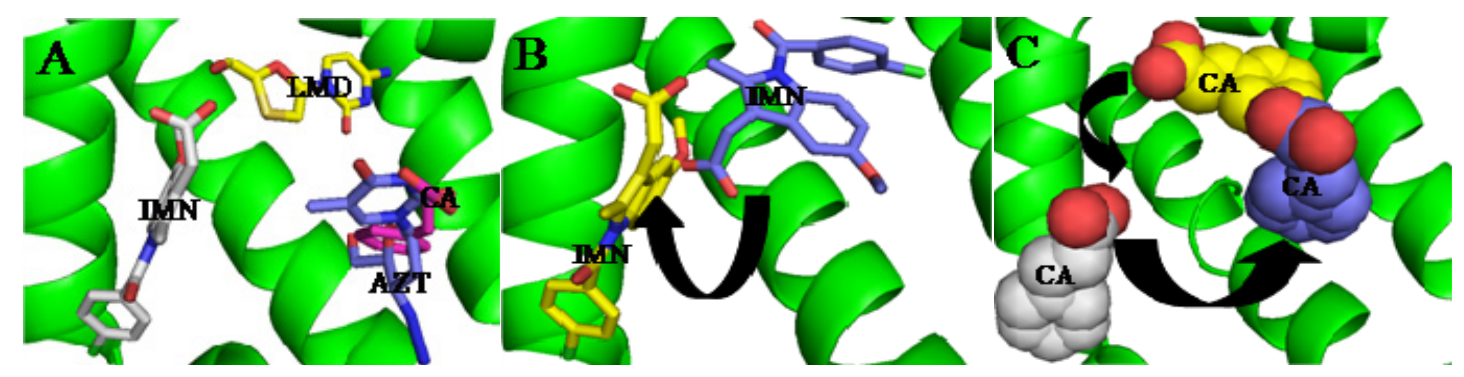

Treatment of brain disease is difficult to date because the majority of drugs cannot pass through the blood brain barrier, which hinders the drugs to reach their target and be clinically efficient [84-86]. Therefore, HSA delivery systems have been extensively studied for treating brain disease since HSA can pass through the blood brain barrier [87-92]. Disappointingly, HSA prefers to bind anionic but not 
cationic drugs in the IIA subdomain [93,94]. The structure of the complex HSA-lidocaine revealed that the drug binds to a unique site formed by residues from subdomain IB facing the central interdomain crevice [67]. The binding is mainly the result of cation- $\pi$ interactions with $\operatorname{Arg} 114$, polar interaction with Lys190, and electrostatic attraction to Asp187 [67]. The IIA subdomain is a big hydrophobic binding site that can hold several drugs at the same time in theory. It raises the tempting possibility that we might be able to change the binding environment of the IIA subdomain to regulate the binding of cationic drugs which could be achieve by using other anionic drugs or by mutating nearby residues [73]. Yang et al. proved that these ideas are feasible. Fluorescence experiments demonstrated that a cationic drug, amantadine hydrochloride (AH), cannot bind the IIA subdomain when the molar ratio of FA to HSA is less than 1:7, but when the molar ratio of FA to HSA is at least 1:8 [73]. To explain the regulatory mechanism that describes the binding of $\mathrm{AH}$ to the IIA subdomain in presence of FA, the structure of the complex HSA-FA-AH was resolved (Figure 5A). The analysis of this structure revealed that one fatty acid (FA7) rotates about $90^{\circ}$ and moves toward the bottom of binding cavity, which makes space to hold another fatty acid (FA8, Figure 5B). When both fatty acids are bound to the IIA subdomain, the binding of $\mathrm{AH}$ is stabilized by presence of interactions of the carboxyl group of FA8 that forms hydrogen bonds with the amino group of AH (Figure 5A). Interestingly, the binding position of $\mathrm{AH}$ overlaps the position where the drugs tend to bind (Figure 5B). Fortunately, the other anionic drugs also stabilize the binding of AH to the IIA subdomain [73]. Therefore, including modification to the IIA subdomain may be a promising strategy to enhance the drug binding and deliver capabilities of HSA especially when we want to redesign HSA to be carrier for drugs that cannot normally bind this protein [95]. These results provide evidence that it is possible to fully exploit the unique binding capacity of HSA's IIA subdomain to achieve the simultaneous delivery of anionic and cationic drugs [73].

Figure 5. (A) Structural basis of AH and two FAs co-binding to subdomain IIA of HAS; (B) Binding mechanism of AH regulated by two FAs in IIA subdomain of HAS. Dashed line (red), hydrogen bond.

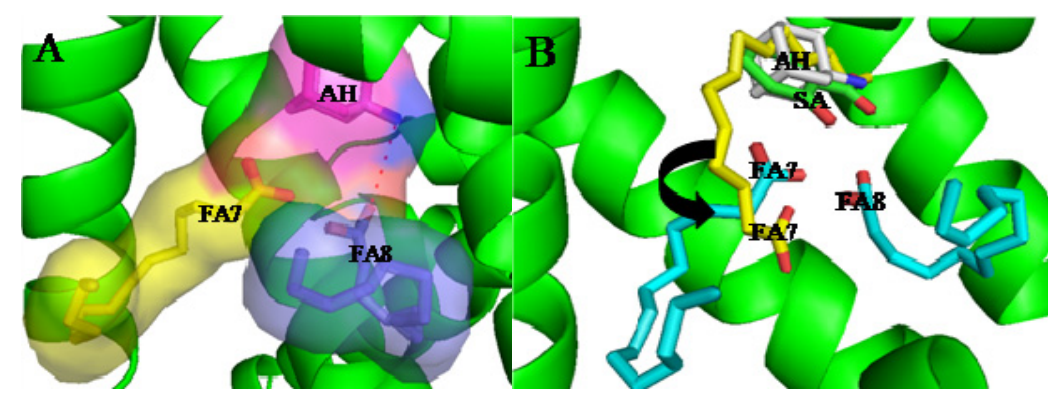

\section{Conclusions}

HSA is a non-toxic and non-antigenic endogenous protein that can carry different hydrophobic and hydrophilic drugs throughout the blood circulation system [95,96]. In addition, the drugs binding with HSA can directly form a "nano-drug" increasing drug bioavailability [2,95]. Thus, HSA-based delivery systems may be one of the most promising drug delivery systems [97-100]. To date, cancer treatment is still a big challenge for humans because anticancer drugs are associated with severe side effects and 
an inconvenient evolution of drug resistance during treatment [101-103]. Therefore, HSA-based delivery systems have been exploited to improve the targeting of anticancer drugs and decrease their side effects [104-107]. Additionally, we can conjugate HSA with active targeting molecules such as aptamers, antibodies, and various over-expressed receptors [108-110]. The ideal carrier should improve drugs efficiency and control the release rate of drugs at the right place [101]. The structural analysis of HSA complexes can guide us in the rational design of this protein by changing its drug binding ability using several strategies including, the modification of compounds' structure, or the regulation of drugs to each other, or edit certain HSA amino acids rendering the HSA carrier more effective for drug delivery to the target site [96]. No doubt, HSA will be more extensively studied in various fields except that it will be more application in medicine field owing to its fascinating properties [95,111-119].

\section{Acknowledgments}

We are grateful to staff of Shanghai Synchrotron Radiation Facility for their technical assistance in data collection. This work was supported by the Natural Science Foundation of China (31060121 and 21171043), Natural Science Foundation of Guangxi (2012GXNSFCB053001, 2013GXNSFGA019010) and Technology division of Guilin (20130403-1).

\section{Conflicts of Interest}

The authors declare no conflict of interest.

\section{References}

1. Peters, T. All about Albumin: Biochemistry, Genetics, and Medical Applications; Academic Press: San Diego, CA, USA, 1996.

2. Fanali, G.; di Masi, A.; Trezza, V.; Marino, M.; Fasano, M.; Ascenzi, P. Human serum albumin: From bench to bedside. Mol. Asp. Med. 2012, 33, 209-290.

3. Fasano, M.; Curry, S.; Terreno, E.; Galliano, M.; Fanali, G.; Narciso, P.; Notari, S.; Ascenzi, P. The extraordinary ligand binding properties of human serum albumin. IUBMB Life 2005, 57, 787-796.

4. Ha, C.E.; Bhagavan, N.V. Novel insights into the pleiotropic effects of human serum albumin in health and disease. Biochim. Biophys. Acta 2013, 1830, 5486-5493.

5. Kragh-Hansen, U.; Chuang, V.T.; Otagiri, M. Practical aspects of the ligand-binding and enzymatic properties of human serum albumin. Biol. Pharm. Bull. 2002, 25, 695-704.

6. Bal, W.; Sokołowska, M.; Kurowska, E.; Faller, P. Binding of transition metal ions to albumin: Sites, affinities and rates. Biochim. Biophys. Acta 2013, 1830, 5444-5455.

7. Yamasaki, K.; Chuang, V.T.; Maruyama, T.; Otagiri, M. Albumin-drug interaction and its clinical implication. Biochim. Biophys. Acta 2013, 1830, 5435-5443.

8. Otagiri, M. A molecular functional study on the interactions of drugs with plasma proteins. Drug Metab. Pharmacokinet. 2005, 20, 309-323. 
9. Ascenzi, P.; Bocedi, A.; Notari, S.; Fanali, G.; Fesce, R.; Fasano, M. Allosteric modulation of drug binding to human serum albumin. Mini Rev. Med. Chem. 2006, 6, 483-489.

10. Bertucci, C.; Domenici, E. Reversible and covalent binding of drugs to human serum albumin: Methodological approaches and physiological relevance. Curr. Med. Chem. 2002, 9, 1463-1481.

11. Colmenarejo, G. In silico prediction of drug-binding strengths to human serum albumin. Med. Res. Rev. 2003, 23, 275-301.

12. Kawakami, A.; Kubota, K.; Yamada, N.; Tagami, U.; Takehana, K.; Sonaka, I.; Suzuki, E.; Hirayama, K. Identification and characterization of oxidized human serum albumin. FEBS J. 2006, 273, 3346-3357.

13. Sleep, D.; Cameron, J.; Evans, L.R. Albumin as a versatile platform for drug half-life extension. Biochim. Biophys. Acta 2013, 1830, 5526-5534.

14. Sugio, S.; Kashima, A.; Mochizuki, S.; Noda, M.; Kobayashi, K. Crystal structure of human serum albumin at $2.5 \AA$ resolution. Protein Eng. 1999, 12, 439-446.

15. Rowland, M.; Tozer, T.N. Clinical Pharmacokinetics and Pharmacodynamics: Concepts and Applications, 4th ed.; Lippincott Williams \& Wilkins: Philadelphia, PA, USA, 2010.

16. Koch-Weser, J.; Sellers, E.M. Binding of drugs to serum albumin (first of two parts). N. Engl. J. Med. 1976, 294, 311-316.

17. Smith, D.A.; Di, L.; Kerns, E.H. The effect of plasma protein binding on in vivo efficacy: Misconceptions in drug discovery. Nat. Rev. Drug Discov. 2010, 9, 929-939.

18. Vallner, J.J. Binding of drugs by albumin and plasma protein. J. Pharm. Sci. 1977, 66, 447-465.

19. Otagiri, M. Study on binding of drug to serum protein. Yakugaku Zasshi 2009, 129, 413-425.

20. Meyer, M.C.; Guttman, D.E. The binding of drugs by plasma proteins. J. Pharm. Sci. 1968, 57, 895-918.

21. Tillement, J.P.; Duché, J.C.; Barré, J. Drug binding to blood proteins: Characteristics, roles and pathophysiological changes. Bull. Acad. Natl. Med. 2006, 190, 935-946.

22. Jusko; W.J.; Gretch, M. Plasma and tissue protein binding of drugs in pharmacokinetics. Drug Metab. Rev. 1976, 5, 43-140.

23. Frostell-Karlsson, A.; Remaeus, A.; Roos, H.; Andersson, K.; Borg, P.; Hämäläinen, M.; Karlsson, R. Biosensor analysis of the interaction between immobilized human serum albumin and drug compounds for prediction of human serum albumin binding levels. J. Med. Chem. 2000, 43, 1986-1992.

24. Ascoli, G.A.; Bertucci, C.; Salvadori, P. Ligand binding to a human serum albumin stationary phase: Use of same-drug competition to discriminate pharmacologically relevant interactions. Biomed. Chromatogr. 1998, 12, 248-254.

25. Watanabe, H.; Tanase, S.; Nakajou, K.; Maruyama, T.; Kragh-Hansen, U.; Otagiri, M. Role of arg-410 and tyr-411 in human serum albumin for ligand binding and esterase-like activity. Biochem. J. 2000, 349, 813-819.

26. Ahmad, B.; Parveen, S.; Khan, R.H. Effect of albumin conformation on the binding of ciprofloxacin to human serum albumin: A novel approach directly assigning binding site. Biomacromolecules 2006, 7, 1350-1356.

27. Sinha, S.S.; Mitra, R.K.; Pal, S.K. Temperature-dependent simultaneous ligand binding in human serum albumin. J. Phys. Chem. B 2008, 112, 4884-4891. 
28. Lucas, L.H.; Price, K.E.; Larive, C.K. Epitope mapping and competitive binding of HSA drug site II ligands by NMR diffusion measurements. J. Am. Chem. Soc. 2004, 126, 14258-14266.

29. Colmenarejo, G.; Alvarez-Pedraglio, A.; Lavandera, J.L. Cheminformatic models to predict binding affinities to human serum albumin. J. Med. Chem. 2001, 44, 4370-4378.

30. Mahesha, H.G.; Singh, S.A.; Srinivasan, N.; Rao, A.G. A spectroscopic study of the interaction of isoflavones with human serum albumin. FEBS J. 2006, 273, 451-467.

31. Garg, A.; Manidhar, D.M.; Gokara, M.; Malleda, C.; Reddy, C.S.; Subramanyam, R. Elucidation of the binding mechanism of coumarin derivatives with human serum albumin. PLoS One 2013, $8, \mathrm{e} 63805$.

32. Abou-Zied, O.K.; AlShihi, O.I. Characterization of subdomain IIA binding site of human serum albumin in its native, unfolded, and refolded states using small molecular probes. J. Am. Chem. Soc. 2008, 130, 10793-10801.

33. Liang, H.; Huang, J.; Tu, C.Q.; Zhang, M.; Zhou, Y.Q.; Shen, P.W. The subsequent effect of interaction between $\mathrm{Co}^{2+}$ and human serum albumin or bovine serum albumin. J. Inorg. Biochem. 2001, 85, 167-171.

34. Shen, X.C.; Liang, H.; Guo, J.H.; Song, C.; He, X.W.; Yuan, Y.Z. Studies on the interaction between $\mathrm{Ag}^{+}$and human serum albumin. J. Inorg. Biochem. 2003, 95, 124-130.

35. Stewart, A.J.; Blindauer, C.A.; Berezenko, S.; Sleep, D.; Sadler, P.J. Interdomain zinc site on human albumin. Proc. Natl. Acad. Sci. USA 2003, 100, 3701-3706.

36. Blindauer, C.A.; Harvey, I.; Bunyan, K.E.; Stewart, A.J.; Sleep, D.; Harrison, D.J.; Berezenko, S.; Sadler, P.J. Structure, properties, and engineering of the major zinc binding site on human albumin. J. Biol. Chem. 2009, 284, 23116-23124.

37. Carter, D.C.; He, X.M.; Munson, S.H.; Twigg, P.D.; Gernert, K.M.; Broom, M.B.; Miller, T.Y. Three-dimensional structure of human serum albumin. Science 1989, 244, 1195-1198.

38. Carter, D.C.; He, X.M. Structure of human serum albumin. Science 1990, 249, 302-303.

39. Carter, D.C.; Ho, J.X. Structure of serum albumin. Adv. Protein Chem. 1994, 45, 153-203.

40. He, X.M.; Carter, D.C. Atomic structure and chemistry of human serum albumin. Nature 1992, 358, 209-215.

41. Curry, S.; Mandelkow, H.; Brick, P.; Franks, N. Crystal structure of human serum albumin complexed with fatty acid reveals an asymmetric distribution of binding sites. Nat. Struct. Biol. 1998, 5, 827-835.

42. Wardell, M.; Wang, Z.; Ho, J.X.; Robert, J.; Ruker, F.; Ruble, J.; Carter, D.C. The atomic structure of human methemalbumin at 1.9 Å. Biochem. Biophys. Res. Commun. 2002, 291, 813819.

43. Wang, Z.M.; Ho, J.X.; Ruble, J.R.; Rose, J.; Rüker, F.; Ellenburg, M.; Murphy, R.; Click, J.; Soistman, E.; Wilkerson, L.; et al. Structural studies of several clinically important oncology drugs in complex with human serum albumin. Biochim. Biophys. Acta 2013, 1830, 5356-5374.

44. Lejon, S.; Frick, I.M.; Björck, L.; Wikström, M.; Svensson, S. Crystal structure and biological implications of a bacterial albumin binding module in complex with human serum albumin. J. Biol. Chem. 2004, 279, 42924-42928. 
45. Lejon, S.; Cramer J.F.; Nordberg, P. Structural basis for the binding of naproxen to human serum albumin in the presence of fatty acids and the GA module. Acta Crystallogr. Sect. F 2008, 64, 64-69.

46. Bhattacharya, A.A.; Curry, S.; Franks, N.P. Binding of the general anesthetics propofol and halothane to human serum albumin. High resolution crystal structures. J. Biol. Chem. 2000, 275, 38731-38738.

47. Zunszain, P.A.; Ghuman, J.; Komatsu, T.; Tsuchida, E.; Curry, S. Crystal structural analysis of human serum albumin complexed with hemin and fatty acid. BMC Struct. Biol. 2003, 3, 6.

48. Ryan, A.J.; Chung, C.W.; Curry, S. Crystallographic analysis reveals the structural basis of the high-affinity binding of iophenoxic acid to human serum albumin. BMC Struct. Biol. 2011, 11, 18.

49. Bhattacharya, A.A.; Grüne, T.; Curry, S. Crystallographic analysis reveals common modes of binding of medium and long-chain fatty acids to human serum albumin. J. Mol. Biol. 2000, 303, 721-732.

50. Petitpas, I.; Grüne, T.; Bhattacharya, A.A.; Curry, S. Crystal structures of human serum albumin complexed with monounsaturated and polyunsaturated fatty acids. J. Mol. Biol. 2001, 314, 955-960.

51. Ryan, A.J.; Ghuman, J.; Zunszain, P.A.; Chung, C.W.; Curry, S. Structural basis of binding of fluorescent, site-specific dansylated amino acids to human serum albumin. J. Struct. Biol. 2011, 174, 84-91.

52. Petitpas, I.; Bhattacharya, A.A.; Twine, S.; East, M.; Curry, S. Crystal structure analysis of warfarin binding to human serum albumin: Anatomy of drug site I. J. Biol. Chem. 2001, 276, 22804-22809.

53. Ghuman, J.; Zunszain, P.A.; Petitpas, I.; Bhattacharya, A.A.; Otagiri, M.; Curry, S. Structural basis of the drug-binding specificity of human serum albumin. J. Mol. Biol. 2005, 353, 38-52.

54. Zunszain, P.A.; Ghuman, J.; McDonagh, A.F.; Curry, S. Crystallographic analysis of human serum albumin complexed with 4Z,15E-bilirubin-IXa. J. Mol. Biol. 2008, 381, 394-406.

55. Petitpas, I.; Petersen, C.E.; Ha, C.E.; Bhattacharya, A.A.; Zunszain, P.A.; Ghuman, J.; Bhagavan, N.V.; Curry, S. Structural basis of albumin-thyroxine interactions and familial dysalbuminemic hyperthyroxinemia. Proc. Natl. Acad. Sci. USA 2003, 100, 6440-6445.

56. Zhu, L.; Yang, F.; Chen, L.; Meehan, E.J.; Huang, M. A new drug binding subsite on human serum albumin and drug-drug interaction studied by X-ray crystallography. J. Struct. Biol. 2008, $162,40-49$.

57. Yang, F.; Bian, C.; Zhu, L.; Zhao, G.; Huang, Z.; Huang, M. Effect of human serum albumin on drug metabolism: Structural evidence of esterase activity of human serum albumin. J. Struct. Biol. 2007, 157, 348-355.

58. Guo, S.; Shi, X.; Yang, F.; Chen, L.; Meehan, E.J.; Bian, C.; Huang, M. Structural basis of transport of lysophospholipids by human serum albumin. Biochem. J. 2009, 423, 23-30.

59. Wang, Y.; Yu, H.; Shi, X.; Luo, Z.; Lin, D.; Huang, M. Structural mechanism of ring-opening reaction of glucose by human serum albumin. J. Biol. Chem. 2013, 288, 15980-15987.

60. Luo, Z.; Shi, X.; Hu, Q.; Zhao, B.; Huang, M. Structural evidence of perfluorooctane sulfonate transport by human serum albumin. Chem. Res. Toxicol. 2012, 25, 990-992. 
61. Wang, Y.; Luo, Z.; Shi, X.; Wang, H.; Nie, L.; Huang, M. A fluorescent fatty acid probe, DAUDA, selectively displaces two myristates bound in human serum albumin. Protein Sci. 2011, 20, 2095-2101.

62. Li, M.; Lee, P.; Zhang, Y.; Ma, Z.; Yang, F.; Zhou, Z.; Wu, X.; Liang, H. X-ray crystallographic and fluorometric analysis of the interactions of Rhein to human serum albumin. Chem. Biol. Drug Des. 2014, 83, 167-173.

63. Yang, F.; Ma, Z.Y.; Zhang, Y.; Li, G.Q.; Li, M.; Qin, J.K.; Lockridge, O.; Liang, H. Human serum albumin-based design of a diflunisal prodrug. Eur. J. Pharm. Biopharm. 2013, 84, 549554.

64. Li, M.; McAuley, E.; Zhang, Y.; Kong, L.; Yang, F.; Zhou, Z.; Wu, X.; Liang, H. Comparison of binding characterization of two antiviral drugs to human serum albumin. Chem. Biol. Drug Des. 2013, doi:10.1111/cbdd.12270.

65. Yamaguchi, S.; Aldini, G.; Ito, S.; Morishita, N.; Shibata, T.; Vistoli, G.; Carini, M.; Uchida, K. $\Delta$ 12-prostaglandin $\mathrm{J} 2$ as a product and ligand of human serum albumin: Formation of an unusual covalent adduct at His146. J. Am. Chem. Soc. 2010, 132, 824-832.

66. Buttar, D.; Colclough, N.; Gerhardt, S.; MacFaul, P.A.; Phillips, S.D.; Plowright, A.; Whittamore, P.; Tam, K.; Maskos, K.; Steinbacher, S.; et al. A combined spectroscopic and crystallographic approach to probing drug-human serum albumin interactions. Bioorg. Med. Chem. 2010, 18, 7486-7496.

67. Hein, K.L.; Kragh-Hansen, U.; Morth, J.P.; Jeppesen, M.D.; Otzen, D.; Møller, J.V.; Nissen, P. Crystallographic analysis reveals a unique lidocaine binding site on human serum albumin. J. Struct. Biol. 2010, 171, 353-360.

68. Mao, H.; Hajduk, P.J.; Craig, R.; Bell, R.; Borre, T.; Fesik, S.W. Rational design of diflunisal analogues with reduced affinity for human serum albumin. J. Am. Chem. Soc. 2001, 123, 10429-10435.

69. Mahesh, G.; Rajagopal, S.; et al. Molecular interaction studies of trimethoxy flavone with human serum albumin. PLoS One 2010, 1, 5, e8834.

70. Oltersdorf, T.; Elmore, S.W.; Shoemaker, A.R.; Armstrong, R.C.; Augeri, D.J.; Belli, B.A.; Bruncko, M.; Deckwerth, T.L.; Dinges, J.; Hajduk, P.J.; et al. An inhibitor of Bcl-2 family proteins induces regression of solid tumours. Nature 2005, 435, 677-681.

71. Vallianatou, T.; Lambrinidis, G.; Tsantili-Kakoulidou, A. In silico prediction of human serum albumin binding for drug leads. Expert Opin. Drug Discov. 2013, 8, 583-595.

72. Tesseromatis, C.; Alevizou, A. The role of the protein-binding on the mode of drug action as well the interactions with other drugs. Eur. J. Drug Metab. Pharmacokinet. 2008, 33, 225-230.

73. Yang, F.; Lee, P.; Ma, Z.; Ma, L.; Yang, G.; Wu, X.; Liang, H. Regulation of amantadine hydrochloride binding with IIA subdomain of human serum albumin by fatty acid chains. J. Pharm. Sci. 2013, 102, 84-92.

74. Yang, F.; Yue, J.; Ma, L.; Ma, Z.; Li, M.; Wu, X.; Liang, H. Interactive associations of drug-drug and drug-drug-drug with IIA subdomain of human serum albumin. Mol. Pharm. 2012, 9, 3259-3265.

75. Curry, S. Lessons from the crystallographic analysis of small molecule binding to human serum albumin. Drug Metab. Pharmacokinet. 2009, 24, 342-357. 
76. Curry, S. X-ray Crystallography of Albumin. In Human Serum Albumin-New Insights on Its Structural Dynamics, Functional Impacts and Pharmaceutical Applications; M. Otagiri, Ed.; Sojo University Publishing Center: Kumamoto, Japan, 2011; pp. 1-29.

77. Sudlow, G.; Birkett, D.J.; Wade, D.N. The characterization of two specific drug binding sites on human serum albumin. Mol. Pharmacol. 1975, 11, 824-832.

78. Sudlow, G.; Birkett, D.J.; Wade, D.N. Further characterization of specific drug binding sites on human serum albumin. Mol. Pharmacol. 1976, 12, 1052-1061.

79. Bos, O.J.; Remijn, J.P.; Fischer, M.J.; Wilting, J.; Janssen, L.H. Location and characterization of the warfarin binding site of human serum albumin - A comparative study of two large fragments. Biochem. Pharmacol. 1988, 37, 3905-3909.

80. Bos, O.J.; Fischer, M.J.; Wilting, J.; Janssen, L.H. Drug-binding and other physicochemical properties of a large tryptic and a large peptic fragment of human serum albumin. Biochim. Biophys. Acta 1988, 953, 37-47.

81. Simard, J.R.; Zunszain, P.A.; Hamilton, J.A.; Curry, S. Location of high and low affinity fatty acid binding sites on human serum albumin revealed by NMR drug-competition analysis. J. Mol. Biol. 2006, 361, 336-351.

82. Simard, J.R.; Zunszain, P.A.; Ha, C.E.; Yang, J.S.; Bhagavan, N.V.; Petitpas, I.; Curry, S.; Hamilton, J.A. Locating high-affinity fatty acid-binding sites on albumin by X-ray crystallography and NMR spectroscopy. Proc. Natl. Acad. Sci. USA 2005, 102, 17958-17963.

83. Meijer, D.K.; van der Sluijs, P. The influence of binding to albumin and $\alpha 1$-acid glycoprotein on the clearance of drugs by the liver. Pharm. Weekbl. Sci. 1987, 9, 65-74.

84. Kuwahara, H.; Nishida, Y.; Yokota, T. Blood-brain barrier and Alzheimer's disease. Brain Nerve 2013, 65, 145-151.

85. Sharma, H.S.; Castellani, R.J.; Smith, M.A.; Sharma, A. The blood-brain barrier in Alzheimer's disease: Novel therapeutic targets and nanodrug delivery. Int. Rev. Neurobiol. 2012, 102, 47-90.

86. Banks, W.A. Drug delivery to the brain in Alzheimer's disease: Consideration of the blood-brain barrier. Adv. Drug Deliv. Rev. 2012, 64, 629-639.

87. Chopra, A. Transferrin-Coated Gadolinium-Labeled Human Serum Albumin Nanoparticles. In Molecular Imaging and Contrast Agent Database (MICAD); National Center for Biotechnology Information: Bethesda, MD, USA, 2013.

88. Kang, Y.S.; Pardridge, W.M. Brain delivery of biotin bound to a conjugate of neutral avidin and cationized human albumin. Pharm. Res. 1994, 11, 1257-1264.

89. Bickel, U.; Yoshikawa, T.; Pardridge, W.M. Delivery of peptides and proteins through the blood-brain barrier. Adv. Drug Deliv. Rev. 2001, 46, 247-279.

90. Patel, M.M.; Goyal, B.R.; Bhadada, S.V.; Bhatt, J.S.; Amin, A.F. Getting into the brain: Approaches to enhance brain drug delivery. CNS Drugs 2009, 23, 35-58.

91. Pardridge, W.M. Drug targeting to the brain. Pharm. Res. 2007, 24, 1733-1744.

92. Chuang, V.T.; Kragh-Hansen, U.; Otagiri, M. Pharmaceutical strategies utilizing recombinant human serum albumin. Pharm. Res. 2002, 19, 569-577.

93. Schönfeld, D.L.; Ravelli, R.B.; Mueller, U.; Skerra, A. The 1.8-A crystal structure of $\alpha 1$-acid glycoprotein (orosomucoid) solved by UV RIP reveals the broad drug-binding activity of this human plasma lipocalin. J. Mol. Biol. 2008, 384, 393-405. 
94. Kragh-Hansen, U. Molecular aspects of ligand binding to serum albumin. Pharmacol. Rev. 1981, $33,17-53$.

95. Zsila, F. Subdomain IB is the third major drug binding region of human serum albumin: Toward the three-sites model. Mol. Pharm. 2012, 10, 1668-1682.

96. Liang, H.; Yang, F.; Lee, N.; Wu, X. HSA-based anti-inflammatory therapy: A new and improved approach. Future Med. Chem. 2014, 6, 119-121.

97. Kratz, F. Albumin as a drug carrier: Design of prodrugs, drug conjugates and nanoparticles. J. Control. Release 2008, 132, 171-183.

98. Neumann, E.; Frei, E.; Funk, D.; Becker, M.D.; Schrenk, H.H.; Müller-Ladner, U.; Fiehn, C. Native albumin for targeted drug delivery. Expert Opin. Drug Deliv. 2010, 7, 915-925.

99. Kratz, F.; Elsadek, B. Clinical impact of serum proteins on drug delivery. J. Control. Release 2012, 161, 429-445.

100. Elsadek, B.; Kratz, F. Impact of albumin on drug delivery-New applications on the horizon. J. Control. Release 2012, 157, 4-28.

101. Allen, T.M.; Cullis, P.R. Drug delivery systems: Entering the mainstream. Science 2004, 303, $1818-1822$.

102. Sweta, M.; Prakash, J.J.; Domb, A.J.; Kumar, N. Exploiting EPR in polymer drug conjugate delivery for tumor targeting. Curr. Pharm. Des. 2006, 12, 4785-4796.

103. Kratz, F.; Beyer, U. Serum proteins as drug carriers of anticancer agents: A review. Drug Deliv. 1998, 5, 281-299.

104. Dosio, F.; Brusa, P.; Crosasso, P.; Arpicco, S.; Cattel, L.G. Preparation, characterization and properties in vitro and in vivo of a paclitaxel-albumin conjugate. J. Control. Release 1997, 47, 293-304.

105. Kratz, F.; Mueller-Driver, R.; Hofmann, I.; Drevs, J.; Unger, C. A novel macromolecular prodrug concept exploiting endogenous serum albumin as a drug carrier for cancer chemotherapy. J. Med. Chem. 2000, 43, 1253-1256.

106. Liu, M.; Lim, Z.J.; Gwee, Y.Y.; Levina, A.; Lay, P.A. Characterization of a ruthenium(III)/NAMIA adduct with bovine serum albumin that exhibits a high anti-metastaticactivity. Angew. Chem. Int. Ed. 2010, 49, 1661-1664.

107. Silva, D.; Cortez, C.M.; Silva, C.M.; Missailidis, S. A fluorescent spectroscopy and modelling analysis of anti-heparanase aptamers-serum protein interactions. J. Photochem. Photobiol. B 2013, 127, 68-77.

108. Yewale, C.; Baradia, D.; Vhora, I.; Misra, A. Proteins: Emerging carrier for delivery of cancer therapeutics. Expert Opin. Drug Deliv. 2013, 10, 1429-1448.

109. Son, S.; Song, S.; Lee, S.J.; Min, S.; Kim, S.A.; Yhee, J.Y.; Huh, M.S.; Chan, K.I.; Jeong, S.Y.; Byun, Y.; et al. Self-crosslinked human serum albumin nanocarriers for systemic delivery of polymerized siRNA to tumors. Biomaterials 2013, 34, 9475-9485.

110. Ehrlich, G.K.; Michel, H.; Truitt, T.; Riboulet, W.; Pop-Damkov, P.; Goelzer, P.; Hainzl, D.; Qureshi, F.; Lueckel, B.; Danho, W.; et al. Preparation and characterization of albumin conjugates of a truncated peptide YY analogue for half-life extension. Bioconjug. Chem. 2013, 24, 2015-2024.

111. Vakhrusheva, T.V.; Gusev, A.A.; Gusev, S.A.; Vlasova, II. Albumin reduces thrombogenic potential of single-walled carbon nanotubes. Toxicol. Lett. 2013, 221, 137-145. 
112. Kragh-Hansen, U. Molecular and practical aspects of the enzymatic properties of human serum albumin and of albumin-ligand complexes. Biochim. Biophys. Acta 2013, 1830, 5535-5544.

113. Ge, C.; Du, J.; Zhao, L.; Wang, L.; Liu, Y.; Li, D.; Yang, Y.; Zhou, R.; Zhao, Y.; Chai, Z.; et al. Binding of blood proteins to carbon nanotubes reduces cytotoxicity. Proc. Natl. Acad. Sci. USA 2011, 108, 16968-16973.

114. Komatsu, T.; Qu, X.; Ihara, H.; Fujihara, M.; Azuma, H.; Ikeda, H. Virus trap in human serum albumin nanotube. J. Am. Chem. Soc. 2011, 133, 3246-3248.

115. Qu, X.; Komatsu, T. Molecular capture in protein nanotubes. ACS Nano 2010, 4, 563-573.

116. Komatsu, T.; Nakagawa, A.; Qu, X. Structural and mutagenic approach to create human serum albumin-based oxygen carrier and photosensitizer. Drug Metab. Pharmacokinet. 2009, 24, 287-299.

117. Komatsu, T.; Ohmichi, N.; Zunszain, P.A.; Curry, S.; Tsuchida, E. Dioxygenation of human serum albumin having a prosthetic heme group in a tailor-made heme pocket. J. Am. Chem. Soc. 2004, 126, 14304-14305.

118. Komatsu, T.; Wang, R.M.; Zunszain, P.A.; Curry, S.; Tsuchida, E. Photosensitized reduction of water to hydrogen using human serum albumin complexed with zinc-protoporphyrin IX. J. Am. Chem. Soc. 2006, 128, 16297-16301.

119. Rozga, J.; Piątek, T.; Małkowski, P. Human albumin: Old, new, and emerging applications. Ann. Transplant. 2013, 18, 205-217.

(C) 2014 by the authors; licensee MDPI, Basel, Switzerland. This article is an open access article distributed under the terms and conditions of the Creative Commons Attribution license (http://creativecommons.org/licenses/by/3.0/). 